\title{
ANÁLISIS DE LA CONFIGURACIÓN ÓPTIMA DE ELECTRODOS PARA LA DETECCIÓN DE ATENCIÓN EN TAREA DE IMAGINACIÓN MOTORA
}

\author{
Luis de la Ossa, Javier V. Juan, Mario Ortiz, Eduardo Iáñez, José M. Azorín; \\ Brain-Machine Interface System Lab, Av. Universidad SN, ed. Innova, Elche, España; \\ luis.ossa@goumh.umh.es, javier.juan02@goumh.umh.es, mortiz@umh.es, eianez@umh.es, \\ jm.azorin@umh.es
}

\begin{abstract}
Resumen
El uso de interfaces cerebro máquina (BCIs) supone un importante avance en el control de dispositivos para la rehabilitación de pacientes. Además, la detección de la atención durante la marcha puede ser fundamental a la hora de garantizar la seguridad en el control de estos dispositivos mediante electroencefalogramas (EEG) y evitar activaciones involuntarias. En esta investigación, realizamos un análisis de diferentes configuraciones de los electrodos que componen una BCI, tratando de discernir qué configuraciones detectan la atención de manera más precisa.
\end{abstract}

Palabras clave: Atención, Imaginación Motora, EEG, Electrodos, Rehabilitación.

\section{MOTIVACIÓN}

Una correcta rehabilitación puede ser clave en la recuperación de los pacientes. En casos en los que el paciente haya experimentado una dolencia que implique una disfunción motora, sea parcial o total, del tren inferior, como pueden ser pacientes que hayan sufrido un accidente cerebrovascular o una lesión espinal, las interfaces cerebro máquina suponen un importante aliado.

De manera resumida, una BCI se encarga de leer los pulsos eléctricos que generan las neuronas del usuario (señales EEG) y los envía a una computadora para poder interpretarlos. Dicho de otro modo, BCI puede actuar como mensajero entre el cerebro y los músculos cuando esta comunicación biológica esté dañada, por ejemplo por alguna de las dolencias citadas previamente. El empleo de estas técnicas es capaz de mejorar notablemente el estado de los pacientes, ya que les ayudan a regenerar los patrones neuronales perdidos [2], [10], [14].

Por su parte, el éxito en las rehabilitaciones basadas en BCIs depende de la interpretación que se haga de los registros EEG efectuados. Este artículo se centra en los procesos cuyo objetivo es conseguir diferenciar la imaginación motora del usuario (su intención de caminar) frente al relax (mantener la mente despejada). Existe mucha literatura al respecto [11]; pero, en general, los métodos se basan en "entrenar" un clasificador, es decir, realizar un número de sesiones en las que se marque cuándo se realiza cada tarea mental, y este algoritmo clasificador extraiga características de las señales EEG registradas para que aprenda a diferenciar cuándo se ejecuta cada una de estas tareas. De esta manera, tras el entrenamiento, se puede probar el algoritmo, ahora sin enviarle información de qué tarea se está realizando, y por tanto el clasificador debe averiguarlo únicamente analizando las señales EEG.

No obstante, la correcta distinción entre estos estados mentales no siempre garantiza un uso seguro del BCI. En aquellos casos en los que se emplee algún dispositivo que asista al paciente en su movimiento, como podría ser un exoesqueleto, y en los que este dispositivo esté controlado por el BCI [4], una mala interpretación de los registros EEG podría desencadenar activaciones involuntarias del exoesqueleto, y esto puede ser peligroso, especialmente en pacientes con discapacidades motoras.

Precisamente por este riesgo, en este estudio se ha tenido en cuenta también el concepto de atención [7], [11], es decir, una tercera tarea a analizar en los registros, junto con imaginación motora y relax. Así, en caso de detectarse imaginación motora, pero no atención, el exoesqueleto no se activará.

A su vez, las BCIs basan su funcionamiento en un conjunto de electrodos que se encarga de registrar las señales EEG. De forma general, los equipos para EEG suelen contar o bien con 64 electrodos, o bien con 32. No obstante, la elección de qué electrodos se van a emplear para el análisis de los registros es un aspecto clave para el éxito del algoritmo de clasificación de tareas mentales, debido principalmente a que una mala elección de los elec- 
trodos de trabajo puede incluir señales EEG en el clasificador que presenten poca o nula información específica sobre las tareas que se desea clasificar, de modo que pueden "confundir" al clasificador e inducirlo a error. No obstante, las regiones del cerebro estimuladas (y que por tanto generan señales EEG que contienen información relevante para el clasificador) varían mucho en función de la naturaleza de las tareas mentales realizadas por el usuario [3], [12], [13].

Sin embargo, se ha demostrado que, en lo referente a la atención, una correcta selección de los electrodos de trabajo es capaz de lograr una mejora de los resultados en la clasificación de las tareas [5]. Es por ello que este estudio se ha focalizado en la búsqueda de las configuraciones óptimas de electrodos a la hora de detectar la atención en usuarios de EEG. Una mejora en estos algoritmos permitiría el desarrollo de técnicas de rehabilitación basadas en dispositivos controlados por BCIs más seguras y eficientes.

\section{MATERIALES Y MÉTODOS}

\subsection{Equipamiento}

Respecto a la BCI, se utilizó un sistema inalámbrico de BrainProducts, compuesto por un amplificador actiCHAMP32, un módulo inalámbrico MOVE32, treinta y dos electrodos, y dos gorros para electrodos de diferente tamaño. El software para la toma y procesamiento de datos está programado por nuestro equipo de investigación utilizando la plataforma MatLab.

El gorro de electrodos sigue la distribución del sistema internacional 10-10. Cuatro de los treinta y dos electrodos se colocaron alrededor de los ojos para la reducción de artefactos oculares. Las zonas elegidas para la colocación de electrodos son las indicadas en la figura 1.

\subsection{Protocolo experimental}

En total se registraron las señales cerebrales de 4 sujetos diferentes (W01, W02, W03, W04), a los que se instruía para que realizaran unas tareas determinadas mientras utilizaban una cinta de andar. Por supuesto, todos los sujetos participaron voluntariamente, dando consentimiento informado.

Para cada uno de los registros, se debía repetir dos veces la secuencia de tareas mostrada en la figura 2. Dicha secuencia consistía en una primera tarea de relax, en la que la cinta se encuentra parada, y el sujeto simplemente debía mantener la mente relajada; a continuación, la cinta se ponía en marcha, y comenzaba la tarea de imaginación motora,

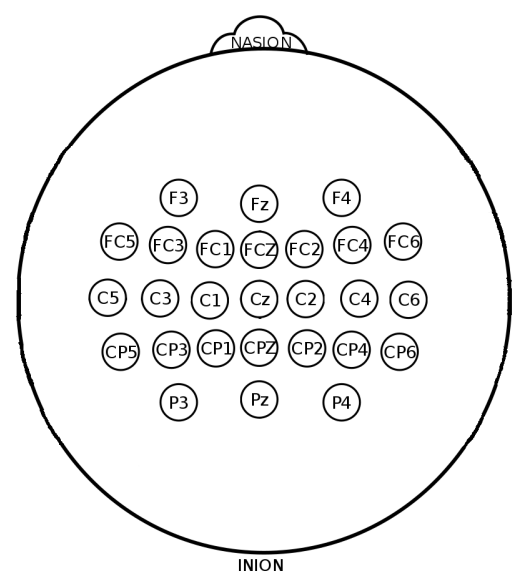

Figura 1: Configuración de los electrodos para los registros

en la que el sujeto debía imaginarse a sí mismo caminando (mientras caminaba); finalmente, el sujeto entraba en la tarea de cuenta regresiva, en la que se le indicaba al sujeto un número de tres dígitos, y otro número del 6 al 9 (que variaba en cada registro) que debía restarle de forma sucesiva mientras que seguía caminando. De esta forma, en el registro de EEG disponíamos de tres estados respecto a la atención en los que se podía encontrar la mente: atención baja, durante el relax; atención alta en la acción de caminar, durante la imaginación motora; y atención alta, pero en una tarea diferente de caminar, durante la cuenta regresiva. Todo este procedimiento fue aprobado por la Oficina de Investigación Responsable de la Universidad Miguel Hernández de Elche, de acuerdo con la declaración de Helsinki para la experimentación con sujetos humanos.

\subsection{Procesamiento de señales}

El algoritmo para la detección de la atención utilizado en esta investigación es el mismo que el publicado en [7], con la diferencia de que en este estudio utilizamos diferentes configuraciones de electrodos. Dicho algoritmo se puede dividir en distintas etapas:

En la primera etapa, el preprocesamiento, se aplica el algoritmo $H^{\infty}[6]$ para reducir los artefactos oculares. Además, los datos originales, tomados con una frecuencia de muestreo de $500 \mathrm{~Hz}$ se remuestreaban a $200 \mathrm{~Hz}$ para reducir la carga computacional. Dicha frecuencia se escogió para mantener las frecuencias de interés de la banda $\gamma(30-$ $90 \mathrm{~Hz}$ ). Finalmente, se aplicaba una normalización a las señales para que los diferentes electrodos tuvieran el mismo peso a la hora de realizar el procesamiento. Dicha normalización es la misma que la aplicada en [1], basada en el parámetro MVT. 


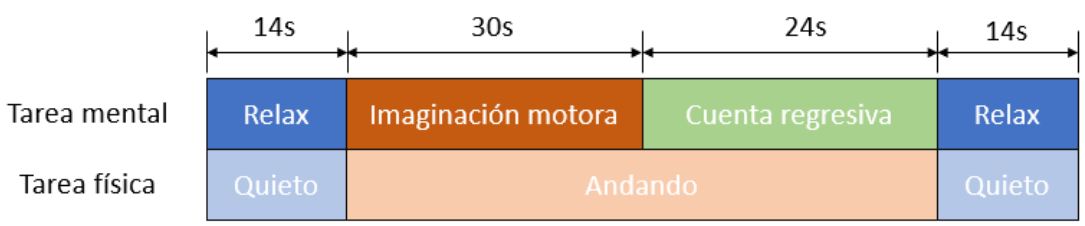

Figura 2: Esquema de las tareas a realizar durante los registros

La siguiente etapa, de procesamiento, comenzaba aplicando un filtro notch de $50 \mathrm{~Hz}$ para filtrar el posible ruido introducido al sistema a través de la red eléctrica. A continuación, se aplicaba un filtro paso banda entre $5-90 \mathrm{~Hz}$, seguido de un filtro de Common Average Reference [8]. Finalmente, como vector de características se utilizaba la densidad espectral de potencia mediante el método de máxima entropía [9], para las bandas entre 30$45 \mathrm{~Hz}$ y $55-90 \mathrm{~Hz}$.

La etapa final corresponde a la clasificación. Para realizar la misma, se empleó un Análisis Discriminante Lineal (LDA, por sus siglas en inglés). Para ello, se consideraban tres clases, cada una correspondiente a una de las tareas: relax, cuenta, e imaginación motora. Este clasificador se aplicaba a cada una de las épocas del registro, y se les asignaba un 0 si eran clasificadas como de la clase "relax" o "cuenta", y un 1 si pertenecían a la clase "imaginación motora". Finalmente, para determinar el nivel de atención de cada época, se realizaba una media aritmética de dicha época y las 9 anteriores. Así, se obtenía un índice entre 0 y 1 que representaba la atención predicha por el algoritmo.

\subsection{Evaluación de configuraciones}

Para evaluar las configuraciones de electrodos, se empleó una división por grupos similar a la encontrada en [7], dividiéndolos según el lóbulo al que pertenecían. Así, el total de electrodos se dividió en 5 grupos: Frontal (F), Fronto-Central (FC), Central (C), Centro-Parietal (CP) y Parietal (P). Además, también se analizaron todas las combinaciones posibles entre dichos grupos, por lo que la cantidad total de configuraciones analizadas asciende a 30 .

Para evaluar cada configuración, se realizaba una validación cruzada, en la que se entrenaba al clasificador con todos los registros de cada sujeto menos uno. A continuación, se aplicaba dicho modelo para predecir el nivel de atención en el registro que no se había utilizado para entrenamiento, obteniendo así una gráfica como la mostrada en la figura 3. Esto se repetía hasta tener las predicciones de la atención de cada uno de los registros de cada sujeto.
Finalmente, para evaluar cada una de las configuraciones estudiadas, se comparaban las predicciones conseguidas con cada grupo de electrodos con las obtenidas utilizando la información de todos los electrodos, obteniendo así una gráfica como la mostrada en la figura 4. Para realizar una comparación objetiva de ambas predicciones, se calculaban las áreas bajo las curvas que formaban durante cada tarea, tanto la predicción con los electrodos específicos como la predicción con todos los electrodos, y se calculaba un índice de acuerdo a la siguiente expresión:

$$
I=\left(\frac{a_{I M}}{A_{I M}}-1\right)+\left(\frac{A_{C}}{a_{C}}-1\right)+\left(\frac{A_{R}}{a_{R}}-1\right)
$$

- $a_{j}$ : Área bajo curva de predicciones realizadas con una configuración de electrodos específica, pertenecientes a la tarea j (Imaginación Motora, Cuenta, Relax)

- $A_{j}$ : Área bajo la curva de predicciones realizadas con todos los electrodos, pertenecientes a la tarea j (Imaginación Motora, Cuenta, Relax)

Así, cada uno de los términos que conforman dicha fórmula evalúa lo mucho que han mejorado o empeorado las predicciones con el grupo de electrodos específicos en comparación con el caso en el que se utilizan todos los electrodos, para cada una de las tareas.

En el caso de la imaginación motora, se busca que el área perteneciente a esta tarea aumente con la elección de electrodos específica respecto al caso en el que se utilizan todos los electrodos. Por tanto, lo deseable es que la relación entre ambas áreas sea mayor que la unidad, y cuanto más alta mejor. Cuando esto se cumpliera, el primer término tendría un valor positivo.

En el caso tanto de la cuenta regresiva como de relax, se busca que el área con el grupo de electrodos específico sea menor que en el caso en el que se usan todos ellos. Por tanto, lo deseable es que las áreas producidas por las curvas de predicciones con todos los electrodos sean mayores que las producidas por los grupos de electrodos específicos. 


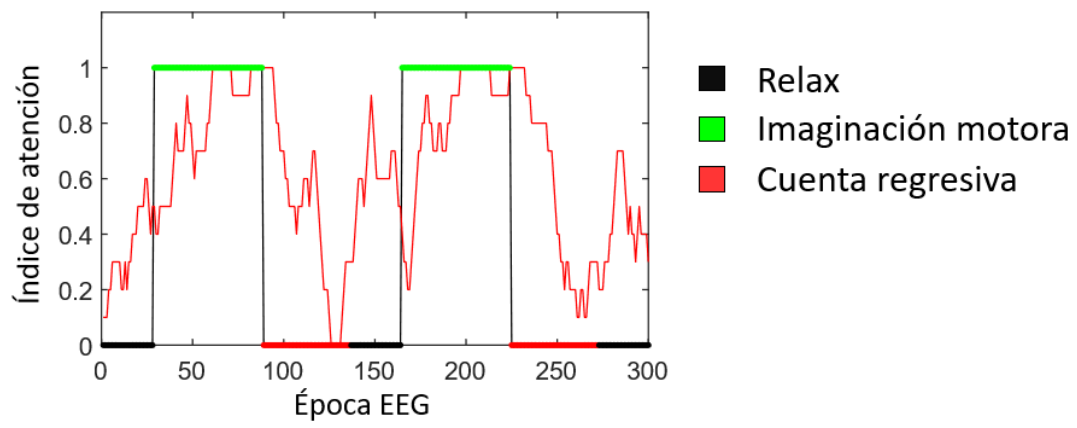

Figura 3: Ejemplo de las predicciones de atención durante uno de los registros.

Cuando esto se cumpliera, el segundo y tercer término también debería tener un valor positivo.

En resumen, cuanto mayor fuera este índice, I, mejor sería la combinación de electrodos analizada para realizar las predicciones de atención.

\section{RESULTADOS}

En la tabla 1 se muestran los índices calculados para cada uno de los sujetos y agrupaciones de electrodos, expresados en porcentaje (multiplicando el índice calculado por 100). Además, en la última columna se muestra la media aritmética de los índices de los cuatro sujetos para cada agrupación. Los cinco mejores índices de cada una de las columnas se encuentran subrayados y en negrita.

Como se puede observar, los resultados empeoraron para la gran mayoría de los casos. Además, la variabilidad entre sujetos es muy elevada. No obstante, sí que es posible detectar algunos casos en los que se mejoró con claridad la detección de la atención, como por ejemplo al emplear únicamente la región frontal (F) del cerebro, en vez de todos los electrodos disponibles, para el caso del sujeto W01. Pese a ello, debido a la ya mencionada variabilidad de los resultados en función del sujeto, no es posible encontrar una configuración que mejore claramente dicha predicción de atención; sin ir más lejos, las predicciones utilizando únicamente la región frontal $(\mathrm{F})$ empeora los resultados en gran medida para todos los otros sujetos. De hecho, dicha selección de electrodos es una de las peores configuraciones para el sujeto W02. Este mismo efecto se puede observar a lo largo de toda la tabla 1, y ninguna de las configuraciones analizadas ha logrado mejorar los resultados de manera global (tomando la media de los resultados con los cuatro sujetos empleados en el estudio) respecto a los logrados con todos los electrodos.

\section{CONCLUSIONES}

Lo que se pretendía con este estudio era analizar la existencia de una selección de electrodos que mejorara la detección de la atención motora en un grupo de sujetos. Sin embargo, como se puede observar en los resultados, no se encontró ninguna configuración que aumentara la predicción de la atención respecto al caso en que se utilizaban todos los electrodos. Sí que se encontraron ciertas configuraciones que funcionaban mejor con unos sujetos que con otros, lo que sugiere la posibilidad de que los mecanismos de atención varíen entre unos individuos y otros. Al fin y al cabo, parece claro que la atención es un fenómeno cognitivo complejo, y su naturaleza parece depender de cada sujeto. Esto sugiere que, quizá, sería positivo realizar un pequeño estudio previo para cada sujeto, y así poder comprobar qué configuraciones funcionan mejor para cada uno, ya que varían entre sujetos, siempre que se vaya a desarrollar alguna aplicación en la que prime la detección de la atención, como las citadas en el primer apartado de este artículo, relacionadas con la rehabilitación.

Otra de las posibilidades podría ser que las características seleccionadas para la clasificación de las señales cerebrales no sean las adecuadas para la detección de este estado mental. Como trabajo futuro, se plantea la realización de nuevas pruebas utilizando características diferentes a la hora de realizar la clasificación. Además, teniendo en cuenta la ya nombrada variabilidad entre sujetos, también planteamos la posibilidad de entrenar una red neuronal convolucional que sea capaz de desarrollar características propias para cada sujeto, lo que mejoraría enormemente la clasificación de este proceso cognitivo. 


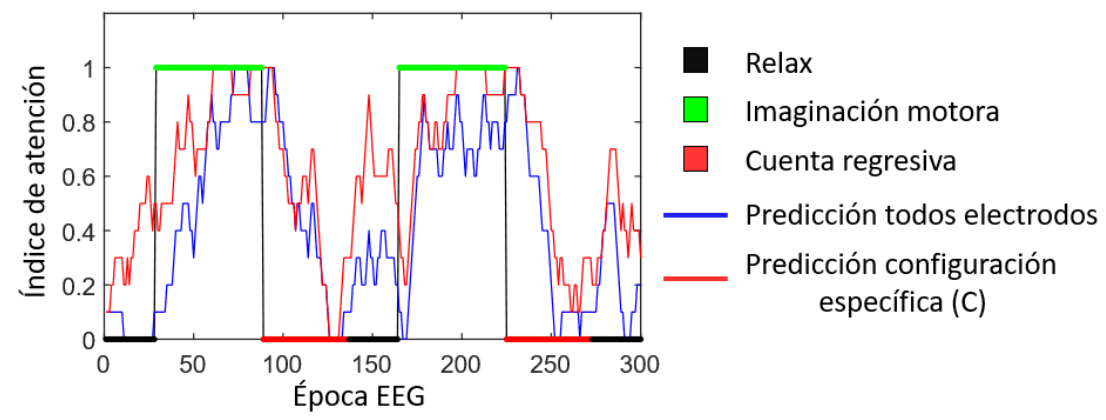

Figura 4: Ejemplo de la evaluación de una de las configuraciones, en la que se utilizaron únicamente electrodos de un grupo $(\mathrm{C})$.

Tabla 1: Resultados finales. Las cuatro primeras columnas muestran los índices calculados, expresados en porcentaje (multiplicados por 100). En la última columna, se muestra la media de los resultados de todos los sujetos. Los mejores índices, tanto por sujeto como de la media total, se encuentran resaltados en negrita y subrayado.

\begin{tabular}{|c|c|c|c|c|c|}
\hline Configuración & W01 [\%] & W02 [\%] & W03 [\%] & W04 [\%] & Media [\%] \\
\hline$F$ & 35 & -115 & -35 & -42 & -39 \\
\hline$F C$ & -6 & -78 & -25 & -50 & -40 \\
\hline$C$ & $\underline{8}$ & $\underline{-3}$ & -25 & -42 & -15 \\
\hline$C P$ & $\underline{-2}$ & -35 & -23 & -42 & -26 \\
\hline$P$ & -14 & -149 & -41 & -32 & -59 \\
\hline$F+F C$ & $\underline{\mathbf{5}}$ & -84 & -24 & -26 & -32 \\
\hline$F+C$ & -24 & $\underline{\mathbf{5}}$ & -25 & -42 & -21 \\
\hline$F+C P$ & -6 & -23 & -14 & -38 & -20 \\
\hline$F+P$ & -32 & -101 & -25 & $\underline{5}$ & -38 \\
\hline$F C+C$ & -25 & -27 & -21 & -38 & -28 \\
\hline$F C+C P$ & -16 & -41 & -8 & -33 & -25 \\
\hline$F C+P$ & -18 & -68 & -13 & -17 & -29 \\
\hline$C+C P$ & -17 & -3 & -11 & -45 & -19 \\
\hline$C+P$ & $\underline{-1}$ & -16 & -12 & -36 & $\underline{-16}$ \\
\hline$C P+P$ & -35 & -19 & -22 & -55 & -33 \\
\hline$F+F C+C$ & -32 & -39 & -16 & -35 & -31 \\
\hline$F+F C+C P$ & -13 & -45 & $\underline{-4}$ & -45 & -27 \\
\hline$F+F C+P$ & -4 & -73 & -11 & -12 & -25 \\
\hline$F+C+C P$ & -16 & $\underline{\mathbf{5}}$ & -9 & -48 & -17 \\
\hline$F+C+P$ & -26 & -10 & -14 & -57 & -27 \\
\hline$F+C P+P$ & -37 & -18 & -12 & -42 & -27 \\
\hline$F C+C+C P$ & -24 & -27 & $\underline{-4}$ & -73 & -32 \\
\hline$F C+C+P$ & -45 & -27 & -16 & -48 & -34 \\
\hline$F C+C P+P$ & -17 & -47 & -5 & -37 & -27 \\
\hline$C+C P+P$ & -17 & $\underline{-1}$ & $\underline{-4}$ & -49 & -18 \\
\hline$F+F C+C+C P$ & -11 & -24 & $\underline{2}$ & -54 & -22 \\
\hline$F+F C+C+P$ & -31 & -12 & -6 & -63 & -28 \\
\hline$F+F C+C P+P$ & -28 & -37 & -8 & $\underline{-26}$ & -25 \\
\hline$F+C+C P+P$ & -17 & $\underline{1}$ & -10 & -45 & -18 \\
\hline$F C+C+C P+P$ & -31 & -13 & $\underline{2}$ & -55 & -24 \\
\hline
\end{tabular}




\section{Agradecimientos}

Esta investigación ha sido realizada en el marco del proyecto DECODED, que ha recibido financiación del Programa de Investigación e Innovación Horizon 2020 de la Unión Europea, mediante una Open Call realizada y ejecutada bajo el proyecto EUROBENCH (grant agreement No 779963).

\section{English summary}

Study of the optimal electrode configuration for attention detection during a motor imagery task

\begin{abstract}
The use of Brain-Machine Interfaces (BMIs) represents a relevant advance in the control of devices destinated to patients rehabilitation. Besides, attention detection during gait becomes vital when guaranteeing a safe control of this devices through electroencephalofraphic signals (EEG), in order to avoid involuntary activations. In this research, we analyze different BMI electrode configurations trying to assure which ones detetcs attention with the higher accuracy.
\end{abstract}

Keywords: Attention, Motor Imagery, EEG, Electrodes, Rehabilitation.

\section{Referencias}

[1] Costa Á, Iáñez E, Úbeda A, Hortal E, Del-Ama AJ, et al. (2016) Decoding the Attentional Demands of Gait through EEG Gamma Band Features. PLOS ONE 11(4): e0154136. https://doi.org/10.1371/journal.pone.0154136

[2] Dimyan MA, Cohen LG. Neuroplasticity in the context of motor rehabilitation after stroke. Nature Reviews Neurology. 2011; 7(2):76-85. doi: 10.1038/nrneurol.2010.200 PMID: 21243015

[3] G. Lantz, R. Grave de Peralta, L. Spinelli, M. Seeck, C.M. Michel. Epileptic source localization with high density EEG: how many electrodes are needed? Clinical Neurophysiology, Volume 114, Issue 1, 2003, Pages 63-69, ISSN 1388-2457.
[4] J. del. R. Millan et al., "Combining braincomputer interfaces and assistive technologies: state-of-the-art and challenges," Frontiers in Neuroscience, vol. 4:161, 2010.

[5] K. Yaomanee, S. Pan-ngum and P. I. N. Ayuthaya, "Brain signal detection methodology for attention training using minimal EEG channels," 2012 Tenth International Conference on ICT and Knowledge Engineering, 2012, pp. 84-89, doi: 10.1109/ICTKE.2012.6408576.

[6] Kilicarslan, A., Grossman, R. G., and Contreras-Vidal, J. L. (2016). A robust adaptive denoising framework for real-time artifact removal in scalp EEG measurements. J. Neural Eng. 13:026013. doi: 10.1088/17412560/13/2/ 026013

[7] Mario Ortiz, Laura Ferrero, Eduardo Iáñez, José M. Azorín, and José L. Contreras- Vidal. Sensory integration in human movement: A new brain-machine interface based on gamma band and attention level for controlling a lower-limb exoskeleton. Frontiers in Bioengineering and Biotechnology, 8:735, 2020.

[8] McFarland, D. J., McCane, L. M., David, S. V., and Wolpaw, J. R. (1997). Spatial filter selection for EEG-based communication. Electroencephalogr. Clin. Neurophysiol. 103, 386-394. doi: 10.1016/S0013-4694(97)000222

[9] Rainford, B. D., and Daniell, G. J. (1994). $\mu \mathrm{SR}$ frequency spectra using the maximum entropy method. Hyperfine Interact. 87, 1129-1134. doi: 10.1007/BF02068515

[10] Stephenson R. A review of neuroplasticity: some implications for physiotherapy in the treatment of lesions of the brain. Physiotherapy. 1993; 79(10):699-704. doi: 10.1016/S0031-9406(10)60008-9

[11] Susan Aliakbaryhosseinabadi, Ernest Nlandu Kamavuako, Ning Jiang, Dario Farina, Natalie Mrachacz-Kersting, Classification of EEG signals to identify variations in attention during motor task execution, Journal of Neuroscience Methods, Volume 284, 2017, Pages 27-34, ISSN 0165-0270.

[12] Van Hoey, G., Vanrumste, B., D'Havé, M. et al. Influence of measurement noise and electrode mislocalisation on EEG dipole-source localisation. Med. Biol. Eng. Comput. 38, 287-296 (2000). 
[13] William Speier, Aniket Deshpande, Nader Pouratian. A method for optimizing EEG electrode number and configuration for signal acquisition in P300 speller systems. Clinical Neurophysiology, Volume 126, Issue 6, 2015, Pages 1171-1177, ISSN 1388-2457.

[14] Zimmerli L, Duschau-Wicke A, Riener R, Mayr A, Lünenburger L. Virtual reality and gait rehabilitation Augmented feedback for the Lokomat. In: Virtual Rehabilitation International Conference, 2009. IEEE; 2009. p. 150-153.

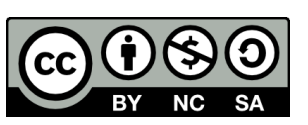

(C) 2021 by the authors. Submitted for possible open access publication under the terms and conditions of the Creative Commons Attribution CC BY-NC-SA 4.0 license (https://creativecommons.org/licenses/by-ncsa/4.0/deed.es). 